\title{
Programa Embu Enxergando Melhor: uma proposta de atenção integral à saúde ocular em pré-escolares
}

\author{
Embu Seeing Better: a complete eye health program proposal for preschool children
}

Maria Cecília S. Lapa ${ }^{1}$, Adriana Mavalli de Freitas², Glaura César Pedroso ${ }^{3}$, Meiry Akiko Furusato4 ${ }^{4}$ Renato Nabas Ventura ${ }^{5}$

\section{RESUMO}

Objetivo: A visão desempenha um papel preponderante no desenvolvimento global da criança. A detecção e o tratamento precoce de problemas visuais são fundamentais para impedir a instalação de seqüelas sensório-motoras irreversíveis. Este estudo visou descrever um programa de atenção integral à saúde ocular de crianças, constituído por ações educativas, de prevenção, de assistência e de reabilitação.

Métodos: Estudo descritivo transversal, no qual se utilizaram dados do treinamento dos professores, da triagem visual realizada na escola e do exame oftalmológico e ortóptico de 23.374 crianças, de cinco a sete anos de idade, matriculadas na rede municipal de educação infantil do município do Embu, no período de 1994 a 2002.

Resultados: Foram capacitados 600 professores e testados $22.118(95 \%)$ pré-escolares. Destes, 4.553 (21\%) precisavam de exame oftalmológico e, deles, 3.016 (66\%) compareceram à consulta. Dos examinados, 1.389 (46\%) receberam prescrição óptica, 477 (16\%), foram encaminhadas para tratamento ortóptico e 1.601 (53\%) receberam alta.

Conclusões: A triagem visual é uma prática eficiente para promover a saúde ocular de pré-escolares, na qual o professor é o elemento chave. Para o êxito destas ações, é fundamental estruturar práticas educativas e de promoção da saúde ocular destinadas às crianças, às famílias e à sociedade.

Palavras-chave: saúde ocular; educação pré-escolar; ação intersetorial; promoção da saúde.

\section{ABSTRACT}

Objective: Early detection and prompt treatment of ocular disorders in children are important to avoid lifelong visual impairment. This study aimed to describe a comprehensive eye health care program for preschool children.

Methods: A descriptive cross-sectional study was carried out focusing eye health conditions of 23,374 children, between five and seven years old, of the public preschool system of Embu, a municipality of Sao Paulo, Brazil, from 1994 to 2002. Data were obtained from preschool vision screening programs performed by teachers and from ocular assessments of all children referred to ophatalmologic and orthoptic evaluation.

Results: 600 preschool teachers qualified for eye problems screening tested 22,118 (95\%) preschool children out of 23,374 that attended the school during the study period. Among them, 4,553 (21\%) needed an ophthalmologic exam and 3,016 (66\%) were examined by the ophthalmologist. Of those examined, 1,389 (46\%) neceived spectacles, 477 $(16.8 \%)$ were referred to orthoptic care and 1,601 (53\%) were discharged.

Conclusions: Visual screening programs are successful for promoting eye health. Teachers, if appropriately trained, are helpful eye care partners. For effective preventive pediatric eye care, it is necessary to promote eye health education programs.

Key-words: eye health; preschool education; intersectorial action; health promotion.
${ }^{1}$ Ortoptista, professora adjunta doutora da Unifesp e coordenadora do programa Embu Enxergando Melhor, São Paulo, SP, Brasil

${ }^{2}$ Oftalmologista da Rede Básica de Saúde do Município de Embu, São Paulo, SP, Brasil

${ }^{3}$ Mestre em Pediatria, pediatra da Universidade Federal de São Paulo (Unifesp) e coordenadora do programa Escola Promotora de Saúde de Embu, São Paulo, SP, Brasil

${ }^{4}$ Ortoptista da Unifesp, preceptora do Curso de Tecnologia Oftálmica da Unifesp e especialista em Educação em Saúde, São Paulo, SP, Brasil

${ }^{5}$ Doutor em Pediatria e pediatra da Unifesp, coordenador do programa de Integração Docente Assistencial Embu/Unifesp, São Paulo, SP, Brasil
Endereço para correspondência:

Maria Cecília S. Lapa

Avenida Piassanguaba, 2.108

CEP: 04060/003

E-mail: mclapa@oftalmo.epm.br

Recebido em: 2/10/2007

Aprovado em: 22/01/2008 


\section{Introdução}

A saúde, entendida durante algum tempo como a ausência de doenças, se deslocou do campo biológico e, hoje, se relaciona às condições de vida da população, necessitando da intervenção direta e intersetorial do poder público, na busca essencial da melhoria da qualidade de vida, com paz, justiça social, eqüidade e cidadania ${ }^{(1)}$.

A promoção da saúde foi definida, em 1986, como o "processo de capacitação da comunidade para atuar na melhoria da sua qualidade de vida e saúde, incluindo uma maior participação no controle deste processo". Assim, a promoção da saúde, além de reorientar as ações do setor, tornou-se um movimento social pelo direito à saúde e pelas condições dignas de vida, calcado nos princípios de eqüidade, intersetorialidade, participação social e solidariedade ${ }^{(2)}$.

A ação intersetorial, na qual o setor de saúde e os demais setores sociais, como educação e meio ambiente, colaboram para o alcance de uma meta comum mediante uma estreita coordenação de suas atribuições, surge como uma nova possibilidade para resolver os problemas que incidem sobre uma população, em um determinado território. O município seria um espaço privilegiado para operacionalizar a intersetorialidade. Num primeiro momento, identificando-se democraticamente os problemas prioritários por todos os setores interessados, tanto da sociedade civil como do poder público e, num segundo momento, efetuando-se um planejamento com todos os sujeitos envolvidos, o qual definirá as tarefas e os responsáveis e garantirá o conhecimento e o reconhecimento de todos neste trabalho. Trata-se, portanto, de um espaço de poder compartilhado e de articulação de interesses, saberes e práticas das diversas organizações participantes ${ }^{(3)}$.

A Escola Promotora de Saúde é uma estratégia adotada para promover, proteger e recuperar a saúde, por possibilitar a ação conjunta de alunos, professores, funcionários e toda a comunidade para melhorar sua qualidade de vida. A escola saudável tem o propósito de contribuir para o desenvolvimento das potencialidades físicas, psíquicas, cognitivas e sociais dos escolares ${ }^{(1)}$. Desta forma, o enfrentamento de problemas complexos de saúde, como a garantia de uma atenção integral à saúde ocular de crianças residentes em um município com alto grau de exclusão social, é de responsabilidade de todas as instâncias governamentais. É preciso superar as propostas centradas no setor da saúde, abrindo perspectivas com novas práticas que contenham verdadeiras ações de interdisciplinaridade e intersetorialidade, permitindo a transformação da realidade local, com melhoria das condições de vida das pessoas e melhoria concreta da saúde ocular das crianças.

A visão desempenha um papel fundamental no desenvolvimento global da criança. As sensações visuais integradas às demais impressões sensoriais constituem o elemento básico para o desenvolvimento da memória, da dedução, de julgamentos de sentimentos e de outras manifestações importantes para o surgimento da inteligência e da personalidade. $\mathrm{O}$ desenvolvimento visual ocorre precocemente e necessita de condições anatômicas e funcionais perfeitas para atingir todo seu potencial. Os primeiros anos de vida são decisivos para obtenção de um sistema visual íntegro ${ }^{(4)}$.

Problemas visuais são freqüentemente encontrados em crianças. Prejudicam o rendimento escolar e podem interferir na própria formação da personalidade. Cerca de 10 a $15 \%$ dos escolares apresentam alterações visuais que incluem: redução da acuidade visual, estrabismo, erro refrativo importante e doenças oculares. Destes, grande parte pode ser prevenida ou minimizada por meio de medidas educativas e assistenciais. Detectar e tratar o mais cedo possível tais distúrbios é fundamental, pois são fatores determinantes para maximizar o potencial visual da criança e impedir a instalação de seqüelas sensório-motoras, por vezes, irreversíveis ${ }^{(4,5)}$.

Programas de triagem visual têm se mostrado efetivos na promoção da saúde ocular e na prevenção, por meio da detecção precoce das alterações visuais. Os professores da pré-escola, pelo acesso diário a um grande contingente de crianças, quando mobilizados e capacitados, se constituem num parceiro chave para estas ações, por meio da aplicação adequada do teste de acuidade visual, do desenvolvimento de programas educativos de higiene visual com pais e alunos e/ou do encaminhamento de crianças que, aparentemente, necessitam de assistência, para serviços de oftalmologia ${ }^{(5-9)}$.

Neste contexto, o presente trabalho teve como objetivo descrever um programa desenvolvido no município do Embu, de atenção integral à saúde ocular de crianças, constituído por ações de educação, prevenção, assistência e reabilitação.

\section{Métodos}

O município do Embu situa-se na porção sudoeste da Região Metropolitana de São Paulo. Conserva um importante centro histórico e turístico, mas a maior parte da população (cerca de 245 mil habitantes) vive em condições de exclusão social, com baixa renda familiar e alto índice de desemprego. O município conta com sete Unidades Básicas de Saúde (UBS), seis Centros de Atenção à Saúde da Família, um 
Centro de Atenção à Saúde do Trabalhador, dois Centros de Atenção Psicossocial, um centro de referência para atenção ao adolescente, dois Prontos-Socorros, uma maternidade municipal e um hospital de referência que atende aos municípios de Embu e Taboão da Serra. Cerca de $40 \%$ da população tem menos de 20 anos de idade e as escolas públicas representam mais de $95 \%$ das matrículas do ensino fundamental e médio ${ }^{(9)}$. Em 2002, 8.710 crianças estavam matriculadas na educação infantil e, destas, 6.980 (80\%) estavam na rede municipal. $\mathrm{Na}$ faixa etária de quatro a seis anos de idade, correspondente a uma população de 13.193 crianças, 6.731 crianças estavam matriculadas na pré-escola, das quais 6.181 $(92 \%)$ na rede municipal ${ }^{(9)}$.

O município possui um programa de saúde escolar desde 1985. Em 2002, foi implantado o programa Escola Promotora de Saúde, com seus três princípios básicos: educação para a saúde com enfoque integral, construção e manutenção de ambientes saudáveis e articulação com os serviços de saúde. O programa Embu Enxergando Melhor foi implantado no município em 1994 e está inserido no Programa de Integração Docente Assistencial, que resultou da parceria entre a Universidade Federal de São Paulo (Unifesp) e o município, iniciada em 1970. Tal parceria tem como objetivo principal a atuação da universidade num sistema regional de saúde, de forma articulada ao poder local e à comunidade.

O programa Embu Enxergando Melhor foi elaborado segundo o princípio da integralidade da atenção à criança e segundo a estratégia Escola Promotora de Saúde. Compreende ações de promoção da saúde ocular, prevenção por meio da detecção precoce de alterações visuais, acompanhamento e tratamento dos danos, incluindo a reabilitação ortóptica dos pré-escolares. Desenvolve-se de forma intersetorial por docentes ortoptistas e estudantes do Curso de Tecnologia Oftálmica da Unifesp, oftalmologista e pediatras da rede Básica de Saúde de Embu, técnicos e professores da Secretaria Municipal da Educação, além de técnicos da Secretaria Municipal de Cidadania e Promoção Social.

O programa mobiliza e capacita, anualmente, os professores da rede municipal do ensino infantil para realizar de triagem visual segura e efetiva. $\mathrm{O}$ treinamento dos professores e coordenadores é feito por meio de oficinas realizadas no início de cada ano, que abordam diferentes temas, como a importância da visão no desempenho global do ser humano e no rendimento escolar; aspectos do desenvolvimento visual normal; noções sobre as doenças visuais comuns nesta faixa etária; importância da detecção e do tratamento precoce das alterações; noções de higiene visual e importância da preservação da visão durante a vida, entre outros. Numa segunda etapa, os participantes recebem treinamento teóricoprático individual e em grupo, voltado exclusivamente ao emprego da tabela de optotipos de Snellen e à medida da acuidade visual, além de material didático informativo a ser utilizado com as crianças, com os profissionais da escola e com as famílias. Ao final do encontro, faz-se uma avaliação do desempenho do programa no ano anterior.

O exame realizado pelo professor, nas crianças de cinco a sete anos de idade, consta da medida da acuidade visual por meio da tabela de optotipos de Snellen, considerada pela literatura um instrumental de referência simples e específico ${ }^{(7,8,10,11)}$, complementada pela observação de sinais e sintomas indicativos de problemas visuais. Os achados são anotados em planilha específica. Todas as crianças com suspeita de alterações visuais que persistem após a retestagem (realizada por outro professor ou profissional capacitado) são encaminhadas para exame médico-oftalmológico completo.

O exame oftalmológico completo é efetuado nas crianças com acuidade visual reduzida para idade e igual ou inferior a 0,7 da tabela utilizada ${ }^{(7)}$ em um ou em ambos os olhos, além daquelas portadoras de sinais, sintomas e comportamento sugestivo de distúrbios oculares como: lacrimejamento, estrabismo, olhos vermelhos, terçóis freqüentes, pálpebras avermelhadas ou com crostas, piscamento excessivo, entre outros achados observados em classe pelo professor e anotados na planilha. As vagas para avaliação oftalmológica, com data e horário pré-fixados, são disponibilizadas para todas as crianças selecionadas.

O oftalmologista da Rede Básica de Saúde de Embu envolvido com o programa se responsabiliza pela promoção, realização/orientação do tratamento das crianças que, após avaliação e diagnóstico, necessitam de cuidado especializado. Os pré-escolares recebem orientação para higiene visual adequada, prescrição óptica sempre que necessário e/ ou encaminhamento para acompanhamento ou tratamento ortóptico, realizado em serviço instituído no município em 1995, exclusivamente para dar suporte terapêutico complementar a estas crianças. As crianças cujo exame especializado não revela anormalidades são orientadas e têm alta.

O professor recebe a informação do resultado do exame oftalmológico e a conduta de todas as crianças que compareceram ao exame, por meio de um formulário de referência e contra-referência utilizado pelo programa Escola Promotora de Saúde no município de Embu.

No contexto deste programa, realizou-se um estudo transversal descritivo, no qual foram utilizados os dados secundá- 
rios das crianças de cinco a sete anos de idade matriculadas na rede municipal de ensino infantil, que participaram do programa Embu Enxergando Melhor, durante o período de 1994 a 2002. Os dados foram obtidos nas oficinas de capacitação dos professores e coordenadores, na triagem visual realizada pelo professor, nos prontuários das crianças atendidas para avaliação oftalmológica e ortóptica. O programa e a utilização dos dados para pesquisa foram aprovados pela Coordenação Técnica da Secretaria da Saúde do Município de Embu. O projeto de pesquisa foi aprovado pelo Comitê de Ética em Pesquisa da Universidade Federal de São Paulo.

\section{Resultados}

No período de 1994 a 2002, foram mobilizados e capacitados para realizar a triagem visual cerca de 600 professores que integram a rede de escolas municipais de educação infantil de Embu, sendo as oficinas anuais freqüentadas, em média, por 50 novos professores e mais 150 que realizam reciclagem periódica.

Neste período, no município de Embu, foram matriculadas 23.374 crianças no ensino infantil. Destas, 22.118 (95\%) participaram da primeira fase do programa, que consistiu de exame de triagem visual e observação de sinais e sintomas sugestivos de transtornos visuais em pré-escolares, realizada pelos professores capacitados.

Os resultados das triagens foram anotados em planilhas próprias. De posse dos achados, o oftalmologista do programa selecionou 4.553 crianças $(21 \%)$ para exame oftalmológico. Dos 4.553 pré-escolares convocados para exame oftalmológico, 3.016 (66\%) compareceram, enquanto $1.537(34 \%)$ faltaram à consulta. Embora a investigação das causas de absenteísmo não tenha sido objeto deste estudo, os pais, quando questionados pelos professores a respeito do não comparecimento, apontaram principalmente problemas econômicos como: falta de recurso financeiro para o translado à UBS Jardim Pinheirinho - local do exame oftalmológico - situada em um dos extremos do município; impossibilidade da faltar ao trabalho e ausência de um cuidador que pudesse acompanhar a criança ao exame; além da provável falta de sensibilização dos pais e familiares em relação aos problemas de saúde ocular.

Das 3.016 crianças examinadas pelo oftalmologista, 938 (31\%) receberam só prescrição óptica. Outras 451 (15\%), além da prescrição óptica, foram encaminhadas para o atendimento ortóptico. Portanto, foram prescritos óculos para 1.389 crianças (6\% do universo estudado). Foram encaminhadas diretamente para avaliação ortóptica, sem prescrição óptica, 26 crianças (1\%). Finalmente, das 3.016 crianças examinadas, $1.601(53 \%)$ receberam alta por não apresentarem alterações ao exame oftalmológico, mesmo que os dados da planilha do professor sugerissem a presença de transtornos visuais.

Das 477 crianças (16\% das avaliadas pelo oftalmologista) encaminhadas para o tratamento ortóptico, 377 (79\%) compareceram à primeira consulta, resultando em índice de absenteísmo de $21 \%$ - menor do que o observado dentre os convocados para consulta oftalmológica (34\%). Porém, $128(34 \%)$ destas crianças abandonaram o tratamento proposto.

\section{Discussão}

Para que fossem incluídas no programa Embu Enxergando Melhor $95 \%$ das crianças de cinco a sete anos de idade que freqüentam as escolas municipais de educação infantil, a atuação dos educadores foi fundamental. A capacitação e mobilização destes profissionais permitiram a realização de ações de promoção da saúde ocular e prevenção dos agravos nesta importante faixa etária, negligenciada pela maior parte das campanhas que contemplam, em geral, escolares a partir dos sete anos ${ }^{(8,12-14)}$. O absenteísmo observado no exame oftalmológico constituiu uma limitação do programa. Apesar do agendamento prévio da consulta realizado pela escola, $34 \%$ das crianças encaminhadas pelos professores não foram levadas para o atendimento. Alguns autores colocam a preocupação com o número relevante de faltosos aos exames oftalmológicos realizados em campanhas e apontam as causas socioeconômicas, a falta de maior divulgação dos programas e de sensibilização dos pais e familiares e, até mesmo, o fato do aluno estar protegido por convênio médico como as principais barreiras ao atendimento dos escolares ${ }^{(15,16)}$.

A efetividade destes programas sempre preocupou todos os que se envolvem com o assunto. Vários são os aspectos apontados na literatura como responsáveis por este questionamento e não diferem, em geral, daqueles enfrentados neste estudo $^{(12,13,17)}$. Neste sentido, a triagem feita pelos professores, no presente estudo, apresentou um alto índice de falsospositivos, indicando problemas relacionados ao aplicador (falha na capacitação), às crianças, ao próprio teste utilizado e ao ambiente onde se realiza a atividade, os quais necessitam ser avaliados e reparados durante a continuidade do programa. Mesmo com estes problemas, a aplicação da triagem pelo professor apresentou uma relação custo/benefício apropriada, pois 
permitiu a inclusão de crianças de cinco a sete anos de idade matriculadas nas escolas municipais de educação infantil do Embu no programa, bem como a detecção de um grande número delas que necessitava de cuidados relacionados à higiene visual, prescrição óptica e reabilitação.

A discordância observada entre os pré-escolares que compareceram à consulta e que realmente necessitaram de cuidados específicos e aqueles que receberam alta (falsospositivos) chama atenção para os instrumentos empregados e para a necessidade de aprimoramento e maior envolvimento dos triadores. A oficina realizada anualmente com os professores, orientadores e diretores de escola visa intensificar o envolvimento do grupo, valorizando a importância da sua participação e do seu trabalho. A oficina reforça os procedimentos, esclarece possíveis dúvidas, discute os pontos falhos e possíveis soluções para as limitações constatadas, mas a equipe ainda busca a estratégia mais adequada para melhorar estes resultados. A literatura ${ }^{(7,12,13,17,18)}$ considera altamente válido o papel do professor como triador, desde que receba treinamento adequado e reforço periódico. Ressalta-se a importância dos possíveis comportamentos do aluno durante a testagem, como simulação, falta de atenção e timidez. Por se tratar de um exame subjetivo, qualquer que seja o procedimento empregado nesta faixa etária, este depende da informação da criança, sendo relevante o fator psicológico. Assim, a medida da acuidade visual nem sempre leva ao mesmo resultado, mesmo quando realizada nas melhores condições.

Neste programa, a baixa condição socioeconômica aparece como fator limitante no que diz respeito à aquisição dos óculos, aspecto fundamental para resolver a dificuldade visual mais comum entre as crianças encaminhadas: a correção de erros refrativos, presentes em cerca de 10 a $15 \%$ dos escolares nesta faixa etária e em torno de $6,4 \%$ na população estudada ${ }^{(12,13)}$. Durante um pequeno período, no início do programa, houve distribuição de óculos pelo Fundo Social do município de Embu; posteriormente, tal medida ficou restrita a casos especiais, após seleção socioeconômica, pois não faz parte dos objetivos do programa. Foram propostas soluções alternativas para o problema, como estimular a concorrência entre as ópticas da região, envolvendo-as nesta problemática e tentando melhores preços. Organizou-se tam- bém um banco de armações em bom estado de conservação, que podem ser reutilizadas.

Em busca da efetividade do programa, foi criado ainda, no próprio município, o Serviço de Ortóptica para prestar atendimento às crianças que, além dos óculos, necessitam de cuidados complementares, como tratamento oclusivo, acompanhamento pré- ou pós-cirúrgico para correção de estrabismo ou determinadas modalidades de exercícios ortópticos. Ainda assim, alguns casos de maior complexidade são encaminhados, com garantia de atendimento, para outros serviços especializados do SUS, em São Paulo. O índice de absenteísmo à primeira consulta, dentre os 477 pré-escolares encaminhados para avaliação ortóptica, foi de $21 \%$ e o de abandono foi de $34 \%$, semelhante ao de outro programa no mesmo município, relacionado a crianças portadoras de doença crônica ${ }^{(17)}$.

A não aderência ao tratamento e a falta de dados precisos sobre o resultado final do tratamento precoce dos problemas visuais, ou seja, a pouca informação a respeito da real diminuição da incidência de ambliopia ou de outras alterações na fase escolar posterior são os grandes desafios da área ${ }^{(14,18-21)}$.

Muitas são as limitações na execução do programa, uma vez que envolve uma cadeia de ações intersetoriais que necessitam da colaboração paritária de todo o grupo. Além disso, a integração efetiva dos professores, da comunidade em geral, dos familiares e de profissionais diversos da área da saúde é fundamental para o êxito das ações preventivas e de promoção à saúde. É preciso formar uma cultura preventiva e de promoção da saúde com a colaboração participativa de todo o grupo que cerca a criança, incluindo os profissionais do setor da saúde, que têm um papel importante, muitas vezes até decisivo, no uso dos óculos e ou da oclusão. Estes, por desconhecimento total do significado e da importância do tratamento, algumas vezes desqualificam o trabalho, comprometendo a possibilidade de recuperar a visão de uma criança que necessita apenas de óculos e/ou de oclusão para solucionar seu problema. Cabe à escola, à família e ao serviço de saúde trabalhar pela inclusão da criança e pelo respeito e apoio do grupo social. Todos os participantes têm papel de destaque e responsabilidade nesta cadeia composta por práticas integradas e interdependentes. 


\section{Referências bibliográficas}

1. Harada J, Pedroso GC, Matos RM, Machado LB. Rede de escolas promotoras de saúde no contexto sociocultural do Município de Embu (SP). In: Brasil Ministério da Saúde, editor. Escolas promotoras de saúde: experiências do Brasil. Brasília: Ministério da Saúde; 2006. p. 69-79.

2. Junqueira LAP. Intersetorialidade, transetorialidade e redes sociais na saúde. Rev Adm Pública 2000;34:35-45.

3. Teixeira CF, Paim JS. Planejamento e programação de ações intersetoriais para a promoção da saúde e da qualidade de vida. Rev Adm Pública 2000;34:63-80.

4. Wright KW. Visual development, amblyopia, and sensory adaptations. In: Wright KW, Buckley EG, Del Monte MA, Ellis FD, Ellis Jr. GS, Mets MB, Stone EM, editors. Pediatric ophthalmology and strabismus. Saint Louis: Mosby; 1995. p. 119-58.

5. Alves MR, Kara-José N, editores. O olho e a visão: o que fazer pela saúde ocular das nossas crianças. São Paulo: Vozes; 1996.

6. Ciner EB, Dobson V, Schmidt PP, Allen D, Cyert L, Maguire M et al. Survey of vision screening policy of preschool children in the United States. Surv Ophthalmol 1999;43:445-57.

7. Temporini ER. Aspectos do plano de oftalmologia sanitária escolar do Estado de São Paulo. Rev Saude Publica 1982;6:243-60.

8. Granzoto JA, Ostermann CSPE, Brum LF, Pereira PG, Granzoto T. Avaliação da acuidade visual em escolares da $1^{\text {a }}$ série do ensino fundamental. Arq Bras Oftalmol 2003;66:167-71.

9. Seade - Fundação Sistema Estadual de Análise de Dados [homepage on the Internet]. Informações dos municípios paulistas [cited 2007 Jul 10]. Available from: http://www.seade.gov.br

10. Lim HC, Quah BL, Balakrishnan V, Lim HC, Tay V, Emmanuel SC. Vision screening of 4-years-old children in Singapore. Singapore Med J 2000;41:271-8

11. Bois C, Binot MC, Jonqua F, Guillemot G, Brémond-Gignac D. Visual screening in 3- to 5-year-old preschool children: Maternal and Infant Welfare evaluation program in the Hauts-de-Seine district, France. J Fr Ophtalmol 2007;30:570-6.

12. Cerqueira E, Cason, EPS, Bastos LFG, Faustino NC, editores. Oftalmologia sanitária escolar. aspectos educativos. São Paulo: Secretaria de Estado dos Negócios da Educação; 1974.

13. Kara-José N, Gonçalves ER, Carvalho RS, editores. Olho no olho. Campanha nacional de prevenção à cegueira e reabilitação visual do escolar. Rio de Janeiro: Cultura Médica; 2006.

14. Gianini RJ, Masi E, Coelho EC, Oréfice FR, Moraes RA. Prevalência de baixa acuidade visual em escolares da rede pública, Sorocaba. Rev Saude Publica 2004;30:201-8.

15. Abud AB, Ottaiano JAA. Aspectos socioeconômicos que influenciam no comparecimento ao exame oftalmológico de escolares com alterações visuais. Arq Bras Oftalmol 2004;67:773-9.

16. Carvalho RS, Temporini ER, Kara-José N. Assessment of visual health campaign activities at schools-teachers' perception. Arq Bras Oftalmol 2007;70:239-45.

17. Ventura RN, Naspitz C, Puccini RF, Silva EMK. Avaliação do programa de atenção a crianças asmáticas acompanhadas nas unidades da saúde do município do Embu, São Paulo, no período de 1988 a 1993. Cad Saude Publica 1998;14:117-28.

18. Russ HHA, Temporini ER, Kara-José N. Impacto da campanha olho no olho em escolas de ensino fundamental - percepção do pessoal de ensino. Arq Bras Oftalmol 2004;67:311-21.

19. Newman DK, East MM. Preschool vision screening: negative predictive value for amblyopia. Br J Ophthalmol 1999;83:676-79.

20. Robinson B, Bobier WR, Martin E, Bryant L. Measurement of the validity of a preschool vision screening program. Am J Public Health 1999;89:193-8.

21. Williamson TH, Andrews R, Dutton GN, Murray G, Graham N. Assessment of an inner city visual screening programme for preschool children. $\mathrm{Br} \mathrm{J}$ Ophthalmol 1995;79:1068-73. 